\title{
Electro-thermally Activated Polymeric Stack for Linear In-plane Actuation
}

\author{
G. K. Lau, J. F. L. Goosen and F. van Keulen \\ Dept. PME, Faculty of $3 \mathrm{ME}$ \\ Delft University of Technology \\ Delft, the Netherlands \\ E-mail: g.k.lau@tudelft.nl
}

\author{
T. Chu Duc and P. M. Sarro \\ Laboratory of ECTM, DIMES \\ Delft University of Technology \\ Delft, the Netherlands
}

\begin{abstract}
An electrothermally-activated polymeric stacked actuator is presented to generate a rectilinear in-plane actuation. The actuator has a novel composite design, comprising of SU8 thermal expandable polymer, a silicon heat conductor and a top thin-film aluminium heater. The silicon heat conductor has a symmetric meandering shape. It extends through a thick SU8 layer. Theory and numerical analyses shows that this design offers advantages of enhanced longitudinal thermal expansion and enhanced heat transfer across thickness of the insulating SU8 layer. It is effective in electromechanical actuation. An activated 530-micron long micro-machined thermal stack demonstrates a longitudinal stroke of 13 microns at $2 \mathrm{~V}$ and $27 \mathrm{~mW}$, and a low temperature rise below $300{ }^{\circ} \mathrm{C}$. Therefore, it can potentially be used for wider applications where low temperature and power efficiency are concerned.
\end{abstract}

\section{INTRODUCTION}

Thermal actuation has several advantages over electrostatic actuation. It features compactness, a high force, a large displacement and a low driving voltage. Thermal actuation has a wide range of applications for both out-plane and in-plane motion. For example, out-plane thermal actuation is useful for driving ciliary conveyors [1] and optical scanners [2]; whereas in-plane thermal actuation is applied to drive optical attenuators [3] and to align fibers [4]. Besides driving functional micro-devices, the in-plane linear thermal actuation is also indispensable element for an in-situ microscopic material tester with integrated load sensing. For example, arrays of V-shape actuators were used for loading a string of carbon nano tube during a tensile test [5].

Performance of thermal actuation depends very much on the expansion materials. Many are made of silicon or metals. However, the silicon and metals have relatively low coefficient of thermal expansion (CTE) and high thermal conductivity. They are not effective in electro-mechanical conversion. Polymers are known to have high CTE $[1,6]$.

This project is sponsored by Delft Center of Mechatronics and Microsystems (DCMM), The Netherlands.
There are increasing interests in using them as expansion material. For example, large out-of-plane actuation was reported using metallic-coated polymeric bi-layers [1]; inplane actuation using the metallic-coated U-shape polymers was also reported [7-8]. Despite these attempts, polymers materials are electrical and thermal insulators. In addition, a thick layer of them is susceptible to unintended out-plane bending and thus not very suitable for in-plane actuation. The out-plane bending may arise from internal stress gradient during fabrication process. It may also arise from large thermal gradient across the thickness when the insulating polymer layer is heated from the surface coated metallic heater.

To resolve the issues in using polymers for effective inplane actuation, a novel design of thermal actuator is presented in this paper. The new design is a composite stack of vertically layered polymer and silicon, with a top metallic heating film (see Fig. 1). The silicon microstructure is meandering in shape, and it extends through the thick layer of the polymer. It forms an effective heat conductor across the thick insulating polymer, whereas the metallic film on its top forms a resistive heater. In addition, the component parallel plates of the meandering microstructure will enhance in-plane longitudinal thermal expansion and stiffness of the polymer filled in the gap between them.

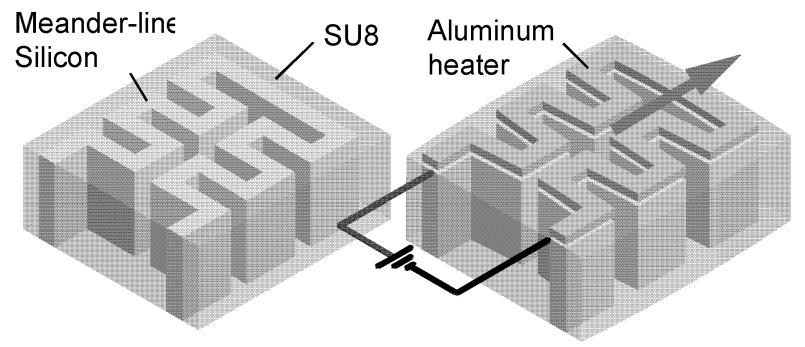

Figure 1. Schematic drawing of the proposed composite thermal actuator: (left) an inactive stack with hidden heating element; (right) an activated stack with deformed shape and actuation direction. 


\section{DESIGN AND ANALYSIS}

\section{A. Design and Driving Principle}

The actuator design consists of a $0.65-\mu \mathrm{m}$ thick aluminum (Al) film, a 50- $\mu \mathrm{m}$ deep silicon ( $\mathrm{Si}$ ) heat conducting microstructure, and a $50-\mu \mathrm{m}$ thick SU8 encapsulating layer (see Fig. 1). The silicon conductor, beneath the film heater, has a meandering layout, symmetric with respect to the longitudinal axis. The meandering structure comprises of many alternating horseshoe bends. Each bend of the conductor structure forms a pair of 30- $\mu \mathrm{m}$ wide, $3-\mu \mathrm{m}$ thick, and vertically standing parallel plates. The $3-\mu \mathrm{m}$ gap and the surrounding of the parallel plates are encased by the SU8 epoxy. The total width of the symmetric meandering structure is $60 \mu \mathrm{m}$. Effectively, the proposed actuator design is a lateral stack of vertically layered Si/SU8 composite, with a continuous path for heat and current flow.

The deep embedded silicon conductor serves to transfer heat from the thin-film heating element to the thermally insulating SU8 polymer. In this way, heat can reach whole layer of the thick polymer, even at the bottom. When heated, the polymer undergoes volumetric expansion to push open the gap between the deep meandering structures, which are basically an array of vertical plates. As the expansion is restrained transversely along the plates, volumetric thermal strain of the composite stack can be accumulated to produce a large longitudinal motion, perpendicular to the plate. In addition, the longitudinal stiffness increases because the polymer strip is restrained from transverse stretching.

\section{B. Theory}

A simplified one-dimensional model of the parallelplated polymer (see Fig. 2) can provide insight into how the longitudinal motion is enhanced. Assuming that the polymer is isotropic and it undergoes small-strain in the linear elastic region, its strain-stress $(\varepsilon-\sigma)$ relationship can be described using a generalized Hooke's law [9]:

$$
\begin{aligned}
& \varepsilon_{11}=\mathrm{C}_{11} \sigma_{11}+\mathrm{C}_{12} \sigma_{22}+\mathrm{C}_{12} \sigma_{33}+\alpha \Delta \mathrm{T} \\
& \varepsilon_{22}=\mathrm{C}_{12} \sigma_{11}+\mathrm{C}_{11} \sigma_{22}+\mathrm{C}_{12} \sigma_{33}+\alpha \Delta \mathrm{T} \\
& \varepsilon_{33}=\mathrm{C}_{12} \sigma_{11}+\mathrm{C}_{12} \sigma_{22}+\mathrm{C}_{11} \sigma_{33}+\alpha \Delta \mathrm{T}
\end{aligned}
$$

where $\alpha$ is CTE, $\Delta \mathrm{T}$ is an uniform temperature rise, and $\mathrm{C}_{\mathrm{ij}}$ are compliance coefficients related to Young's modulus (E) and Poisson's ratio (v): $C_{11}=1 / E, C_{12}=-v / E$

The two parallel plates of the rigid conductor of negligible CTE restrain the polymer strip. Upon heating, thermal strains of the polymer strip are blocked in the transverse direction but allowed in the perpendicular direction. Hence, transverse strains of the polymer are zero: $\varepsilon_{11}=\varepsilon_{22}=0$. As a result, the volumetric thermal strains are

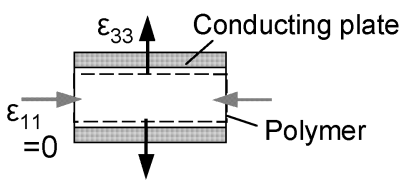

Figure 2. a simplified model of parallel-plated polymer strip, with transverse strain $\varepsilon_{11}$ and perpendicular strain $\varepsilon_{33}$ all accumulated into the perpendicular direction. The simplified model predicts a higher linear constrained thermal strain $\alpha_{\perp}$, which relates to Poisson's ratio $v$ and the unconstrained CTE $\alpha$ :

$$
\alpha_{\perp}=\frac{\varepsilon_{33}}{\Delta T}=\frac{1+v}{1-v} \alpha
$$

The $L$ long composite stack is made of vertical layers of both silicon and polymers. The $\varphi$ fraction for polymer vertical layers produces more significant thermal expansion than the remaining fraction of low CTE silicon. Thus, the total thermal displacement along the longitudinal direction of the stack be approximated by $\alpha_{\perp} \times \varphi L \Delta \mathrm{T}_{\mathrm{avg}}$ where $\Delta \mathrm{T}_{\mathrm{avg}}$ is an average temperature rise.

\section{Material Properties}

Reported thermal and elastic properties of SU8 vary, depending on measurement methods and SU8 deposition processes. Bow test of a SU8 layer on Si substrate measured a bi-axial modulus of $5.18 \mathrm{GPa}$ and CTE of $52 \times 10^{-6} /{ }^{\circ} \mathrm{C}$ at room temperature [6]. Yet, comprehensive tests of SU8 reports transversely isotropic properties: in-plane modulus of $3.2 \mathrm{GPa}$, out-plane modulus of $5.9 \mathrm{GPa}$ at room temperature, in-plane CTE of $87.1 \times 10^{-6} /{ }^{\circ} \mathrm{C}$ and out-plane CTE of 278 $\times 10^{-6} /{ }^{\circ} \mathrm{C}[6]$. In addition to process variation, elastic and thermal properties are temperature and pressure dependent: bulk modulus of SU8 is inversely proportional to temperature while volumetric thermal expansion is inversely proportional to hydrostatic pressure. Despite the variations with respect to temperature, the CTE of SU8 is at least 20 times higher than that of silicon. Thus, the SU8 is suited as expansion material for electro-thermal actuation, with an added advantage of being photo-patternable. Table 1 listed comparison of material properties. The listed thermal and mechanical SU8 properties are in-plane isotropic.

TABLE I. COMPARISON OF THERMAL EXPANDABLE MATERIALS

\begin{tabular}{|l|l|l|l|}
\hline \multirow{2}{*}{\multicolumn{1}{|c|}{ Material Properties }} & \multicolumn{3}{|c|}{ Materials } \\
\cline { 2 - 4 } & \multicolumn{1}{|c|}{$\boldsymbol{A l}[10]$} & \multicolumn{1}{c|}{$\boldsymbol{S i}[11]$} & \multicolumn{1}{c|}{ SU8 $[6]$} \\
\hline Young's modulus $(\mathrm{E}), \mathrm{GPa}$ & 69 & 130 & 3.2 \\
\hline Poisson's ratio $(v)$ & 0.35 & 0.28 & 0.33 \\
\hline Linear CTE $(\alpha), 10^{-6} / \mathrm{K}$ & 23.1 & 2.6 & $87.1 \|$ \\
\hline Electrical resistivity $(\Omega \mathrm{m})$ & $2.7 \times 10^{-8}$ & 0.02 & $>1.2 \times 10^{10}$ \\
\hline Thermal conductivity $(\mathrm{W} / \mathrm{m} / \mathrm{K})$ & 237 & 148 & 0.2 \\
\hline Temperature limit $\left({ }^{\circ} \mathrm{C}\right){ }^{\mathrm{a}}$ & $660\left(\mathrm{~T}_{\mathrm{m}}\right)$ & $1414\left(\mathrm{~T}_{\mathrm{m}}\right)$ & $238\left(\mathrm{~T}_{\mathrm{g}}\right)$ \\
\hline
\end{tabular}

\section{Numerical Simulation}

Finite element method is used to simulate the electrothermal-mechanical response of the actuator. To simplify the simulation, anisotropic properties across the thickness are ignored. Instead, isotropic in-plane material properties listed in Table 1 are used as input to the simulation. The multiphysics simulation is solved using a commercial finite 
element package (ANSYS), based on a model of coupledfield elements (SOLID5). As illustrated in Fig. 3, the root of the thermal stack is fixed with zero displacement and kept at the room temperature of $25^{\circ} \mathrm{C}$. An electric potential is applied across the two ends of the meandering resistor. The steady-state simulation predicts that effective heating throughout a $50-\mu \mathrm{m}$ thick SU8 is possible with the thicknessextended silicon heat conductor. When driven at $1 \mathrm{~V}$, the stack has an average temperature $160{ }^{\circ} \mathrm{C}$ and a peak temperature of $246^{\circ} \mathrm{C}$ at the tip. The simulated resistance at $1 \mathrm{~V}$ is $96 \Omega$, reflecting the average temperature rise.

Tip displacement for the $530 \mu \mathrm{m}$ long stack is simulated to be $4.4 \mu \mathrm{m}$ at $1 \mathrm{~V}$. It works out to have an effective CTE of $60.0 \times 10^{-6} /{ }^{\circ} \mathrm{C}$. Given a longitudinal SU8 fraction of $\varphi=53 \%$, the unconstrained model predicts CTE value of $\varphi \alpha=$ $46.1 \times 10^{-6} /{ }^{\circ} \mathrm{C}$ but the constrained theoretical model predicts CTE upper bound of $\varphi \alpha_{\perp}=91.4 \times 10-6 /{ }^{\circ} \mathrm{C}$. The FEM simulated value is smaller than the theoretical upper limit because FEM accounts for additional stiffness of horseshoe bend, whereas the theoretical model does not. A stack with wider plates is expected to exhibit a higher FEM simulated CTE in approach to the theoretical upper bound as a result of decreasing horseshoe-bend stiffness.

(a)

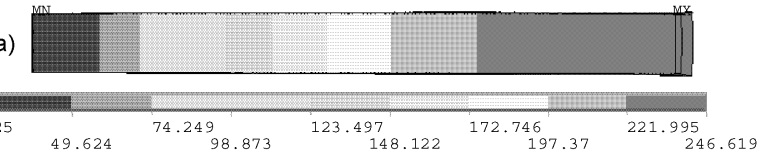

(b)

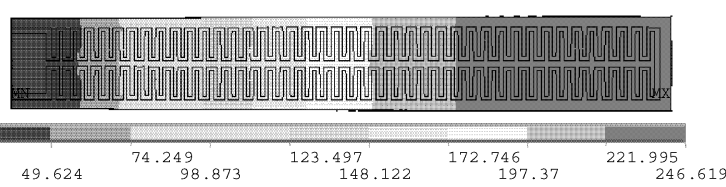

(c)

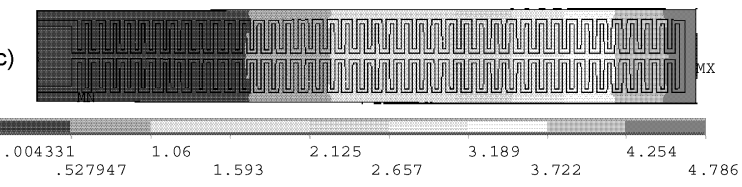

(d)

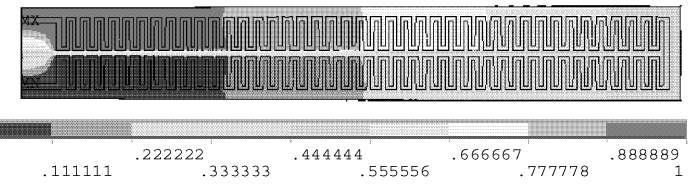

Figure 3. FEM-simulated field contours of a thermal stack, where left side is the root: (1) temperature field on the side; (2) temperature field on the top; (3) longitudinal displacement field on the top; (4) electric potential field on the top.

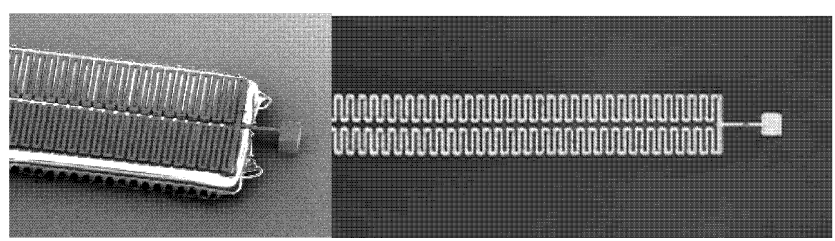

Figure 4. .Pictures of micro-fabricated devices: (left $)$ a SEM micrograph showing a SU8/Si composite stack still residing on top of silicon substrate; (right) an optical image showing a released stack of SU8/Si/Al, with $\mathrm{Al}$ being reflective but SU8 being transparent under optical lighting.

\section{FABRICATION AND TESTING}

\section{A. Fabrication}

The device is fabricated using bulk silicon micromachining and SU8-2002 polymer filling. First, an Al film and a $\mathrm{Si}_{3} \mathrm{~N}_{4}$ film are sputtered, respectively, on the front and the back of a silicon substrate. Thereafter, they are patterned using plasma etching. Then, the silicon is deep etched with the photoresist/aluminum mask on the front side, forming cavities and trenches, surrounding the meandering structure. Afterwards, SU8-2002 is cast and patterned to encase the meandering structure. Finally, the composite structure is released from the backside by $\mathrm{KOH}$ and reactive ion etching (RIE). More details about the fabrication are reported in companion paper [12].

\section{B. Characterization}

Characterization of the micro-actuator is performed on a probe station (Cascade MicroTek) equipped with an optical microscope, using an embedded power supply of a network analyzer (HP/Agilent 8510). A voltage ramp with preset maximum is applied across the contact pads of the aluminum heating film to activate the micro-actuator. Displacement is
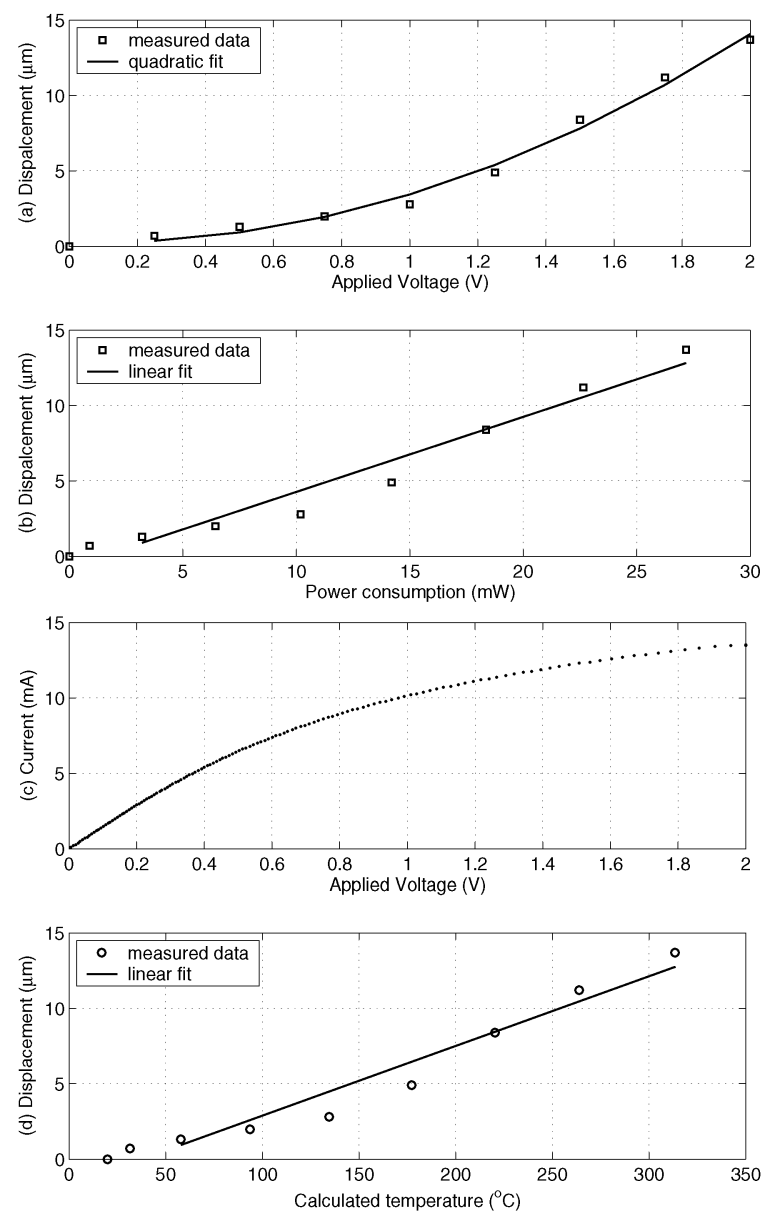

Figure 5. Measurement: (a) measured displacement versus voltage; (b) measured displacement versus power; (c) measured current versus voltage; (d) measured displacement versus calculated temperature 
measured by comparing the magnified optical image of the activated stack reaching steady state at the preset maximum voltage with that before activation. Measurement shows that the tip displacement of the stacked actuator increases almost quadratically with the voltage, while it increases almost linearly with the input power (see Fig. 5a-b).

As the input voltage is ramped up, resistive heating causes a rise in resistance and a drop of the measured current (see Fig. 5c). An average temperature rise along the aluminum heater can be derived from the change in resistance. It is assumed that the rise of resistance is proportional to the temperature rise with a constant temperature coefficient resistance (TCR). Therefore, the average temperature rise is derived as $\Delta \mathrm{T}_{\mathrm{avg}}=$ $\left(\mathrm{R}\left(\mathrm{T}_{\mathrm{avg}}\right)-\mathrm{R}\left(\mathrm{T}_{0}\right)\right) / \mathrm{R}\left(\mathrm{T}_{0}\right) / \mathrm{TCR}$, where the measured $\mathrm{R}\left(\mathrm{T}_{0}\right)$ is $67 \Omega$ and the TCR in use is reported as $4.13 \times 10^{-3} /{ }^{\circ} \mathrm{C}$ [10]. Assuming that the heat dissipation is solely via the conduction to the silicon substrate, the derived average temperature rise is good enough to correlate to or be representative of the temperature rise, which is simulated by FEM to be uniform across the thickness.

The tip of thermal stack is measured to travel $3 \mu \mathrm{m}$ at 1.0 $\mathrm{V}$, and $13 \mu \mathrm{m}$ at $2.0 \mathrm{~V}$. This shows that the newly developed actuator can achieve large actuation at a lower driving voltage. In addition, it is observed that the large displacement at $2.0 \mathrm{~V}$ is only at a power consumption of 27 $\mathrm{mW}$. Based on the derived temperature and the measured displacement (Fig. 4d), the effective CTE is calculated as the slope of linear fit divided by the stack length, i.e. $86 \times 10^{-6} /{ }^{\circ} \mathrm{C}$. If the reported in-plane CTE is true to the tested sample, the measured effective CTE suggests that the constrained polymer is enhanced in longitudinal thermal expansion as compared to the unconstrained. In addition, the constrained polymer is expected to be reinforced with the embedded silicon meandering microstructures.

\section{Potential Applications}

As demonstrated theoretically and experimentally, the newly proposed electro-thermal actuator of polymeric stack is capable of producing a large rectilinear displacement at a low driving power and at a low operating temperature. The effective thermal actuation offers a new opportunity of wider applications, in which conventional designs of thermal actuators are not effective or not capable. Given these enhancement in performances and thermally insulating properties, the new polymeric thermal actuator could even be employed to replace an electrostatic bank of lateral comb drives, in driving a multi-level scanning mirrors [13] or driving a 'battering ram' for measuring surface forces [14].

\section{CONCLUSION}

We have successfully designed, fabricated, and tested a composite stacked actuator of vertically layered SU8 and Si with an $\mathrm{Al}$ heating film. The laterally stacked composite delivered an enhanced in-plane longitudinal thermal strain, using the constrained polymer that out-performs the unconstrained. The $50-\mu \mathrm{m}$ deep and $530-\mu \mathrm{m}$ long polymer stack demonstrated a large displacement of $13 \mu \mathrm{m}$ at $2 \mathrm{~V}$ and at $27 \mathrm{~mW}$. As it is power-efficient, the new design can potentially be used for a wider range of applications, as compared to conventional thermal actuators made of silicon or metals. Potential applications include low-power micropositioning, low-temperature object manipulation, and micro-instruments for material testing.

\section{ACKNOWLEDGMENT}

This project is funded by the Delft Center of Mechatronics and Microsystems (DCMM). The first author would like to acknowledge Dr. K.M.B. Jansen, M. Langelaar and J. Wei, for stimulating discussions. Also, the authors are grateful to the DIMES-ICP group for technical supports.

\section{REFERENCES}

[1] M. Ataka, A. Omodaka, N. Takeshima, and H. Fujita, "Fabrication and operation of polyimide bimorph actuators for a ciliary motion system," J. Microelectromech. Syst., vol. 2, pp. 146 - 150, 1993.

[2] J. P. Yang, X. C. Deng and T. C. Chong, "An electro-thermal bimorph-based microactuator for precise track-positioning of optical disk drives," J. Micromech. Microeng. , vol. 15, pp. 958-965, 2005.

[3] J. C. Chiou, W. T. Lin, "Variable optical attenutor using a thermal actuator array with dual shutters," Opt. Communications, vol. 237, pp. 341-350, 2004

[4] L. A. Field, D. L. Burriesci, P. R. Robrish, and R. C. Ruby, "Micromachined 1x2 optical-fiber switch," Sensors and Actuators APhysical, vol. 53 , pp. 311-315, 1996.

[5] Y. Zhu and H. D. Espinosa, "An electro-mechanical material testing system for in situ electron microscopy and applications," Proc. Natl Acad. Sci., vol. 102, pp. 14503-8, 2005.

[6] R. Feng and R. J. Farris, "The characterization of thermal and elastic constant for an epoxy photoresist SU8 coating," J. Mater. Sci., vol. 27, pp. 4793-4799, 2002.

[7] N.-T. Nguyen, S.-S. Ho and C. L.-N. Low, "A polymeric microgripper with integrated thermal actuators," J. Micromech. Microeng. , vol. 14 pp. 969-974, 2004.

[8] N. Chronis and L. P. Lee, "Electrothermally activated SU-8 microgripper for single cell manipulation in solution," J. Microelectromech. Syst., vol. 14, pp. 857- 863, 2005.

[9] W. M. Lai, D. Rubin and E. Krempl, Introduction to Continuum Mechanics, Butterworth, Heinemann, 1996.

[10] Efunda, "General information on element aluminium," http://www.efunda.com/materials/elements/element_info.cfm?Eleme nt_ID=Al

[11] crystaltechno, "Materials: base properties of silicon," http://www.crystaltechno.com/Materials/Si.htm

[12] T. Chu Duc, G. K. Lau, J. Wei and P. M. Sarro, "Integrated SiliconPolymer Laterally Stacked Bender for Sensing Microgrippers," Proc. $5^{\text {th }}$ IEEE Conference on Sensors, 2006, in press

[13] L. Zhou, J. M. Kahn., and K. S. J. Pister, "Scanning micromirrors Fabricated by an SOI/SOI wafer-bonding process," J. Microelectromech. Syst., vol. 15, pp. 24-32, 2006

[14] W. M. van Spengen, and J. W. M. Frenken, "A 'nano-battering ram' for measureing surface forces,", Proc. 17th MicroMechanics Europe, pp. 205-208, 3-5 September 2006. 